\title{
Le poids des normes dans les phénomènes de l'induction des communications
}

Alex Mucchielli

\section{(2) OpenEdition}

12 Journals

Édition électronique

URL : http://journals.openedition.org/communicationorganisation/2002

DOI : 10.4000/communicationorganisation.2002

ISSN : $1775-3546$

Éditeur

Presses universitaires de Bordeaux

Édition imprimée

Date de publication : 1 novembre 1997

ISSN : 1168-5549

Référence électronique

Alex Mucchielli, «Le poids des normes dans les phénomènes de l'induction des communications », Communication et organisation [En ligne], 12 | 1997, mis en ligne le 26 mars 2012, consulté le 01 mai 2019. URL : http://journals.openedition.org/communicationorganisation/2002 ; DOI : 10.4000/ communicationorganisation.2002

Ce document a été généré automatiquement le 1 mai 2019.

(c) Presses universitaires de Bordeaux 


\title{
Le poids des normes dans les phénomènes de l'induction des communications
}

\author{
Alex Mucchielli
}

1 Je vais montrer qu'une partie des phénomènes dits «d'induction» passent par l'intermédiaire d'éléments du contexte situationnel, eux-mêmes attachés à des normes sociales. On peut donc dire qu'en dernier ressort ce sont les normes interpelées par la situation qui « influencent » les phénomènes de communication. Ces normes sociales, qui agissent sur les échanges entre les acteurs sociaux, sont soit des normes « établies » (des "déjà là » sociaux) qui sont convoquées par certains éléments de la situation dits : "éléments inducteurs"; soit des normes mises en place par les acteurs à travers une organisation de leurs activités; soit encore des normes «émergentes » à travers des communications particulières. Je prendrais une série d'exemples pour illustrer ces trois types d'intervention des normes.

\section{Élément inducteur : proposition de définition}

2 On peut dire, comme en électricité, qu'il y a "induction" lorsque des éléments, interagissant les uns sur les autres, créent un phénomène dit phénomène induit. On peut aussi dire, comme en biologie (ou en psychologie), qu'il y a induction lorsque des éléments "organisateurs» guident un certain développement biologique (ou psychologique). L'induction est donc une action à distance faite par un élément du contexte situationnel sur la communication elle-même.

3 Une « communication » n'est pas analysable en elle-même. On peut s'en rendre compte facilement en constatant qu'aucune description d'une «communication » ne se fait sans qu'il y ait des notations sur les éléments du contexte qui l'accompagnent. Le processus de communication est donc lié à l'ensemble des éléments définissant la situation dans laquelle se déroule la communication. Ces éléments sont de toute nature, ce sont aussi 
bien les intentions des acteurs, les enjeux présents ou encore les caractéristiques physiques et spatiales de la situation de communication. Parmi les éléments constitutifs de la situation de communication, il existe des éléments qui ont ou prennent un relief particulier. Ce sont des éléments qui pèsent sur la situation. Ils ont un statut particulier du fait de leur influence, plus forte que d'autres influences.

Les influences des éléments situationnels inducteurs sont donc fondamentalement liées à leurs « significativités » conscientes ou non pour les acteurs. À la limite, un élément qui "n'induit rien » chez les acteurs n'est même pas «perçu », il est fondu dans le décor général. Chaque situation de communication peut alors être schématisée à partir de quelques éléments « forts » qui s'organisent alors en système. Ces éléments inducteurs se détectent à travers les réponses aux questions: "qu'est-ce qui est prégnant?", ou, « qu'est-ce qui est réellement significatif pour les acteurs, dans cette situation? ». Il s'agit de repérer le ou les « détails » qui «font sens », qui révèlent, par leur présence, tout un arrière-plan d'autres situations sociales.

5 Voyons ceci sur un exemple de simulation de communication à travers les réponses obtenues à un test projectif.

6 Quel est le sens de la situation appréhendée par des individus que l'on met devant le dessin ci-dessous?

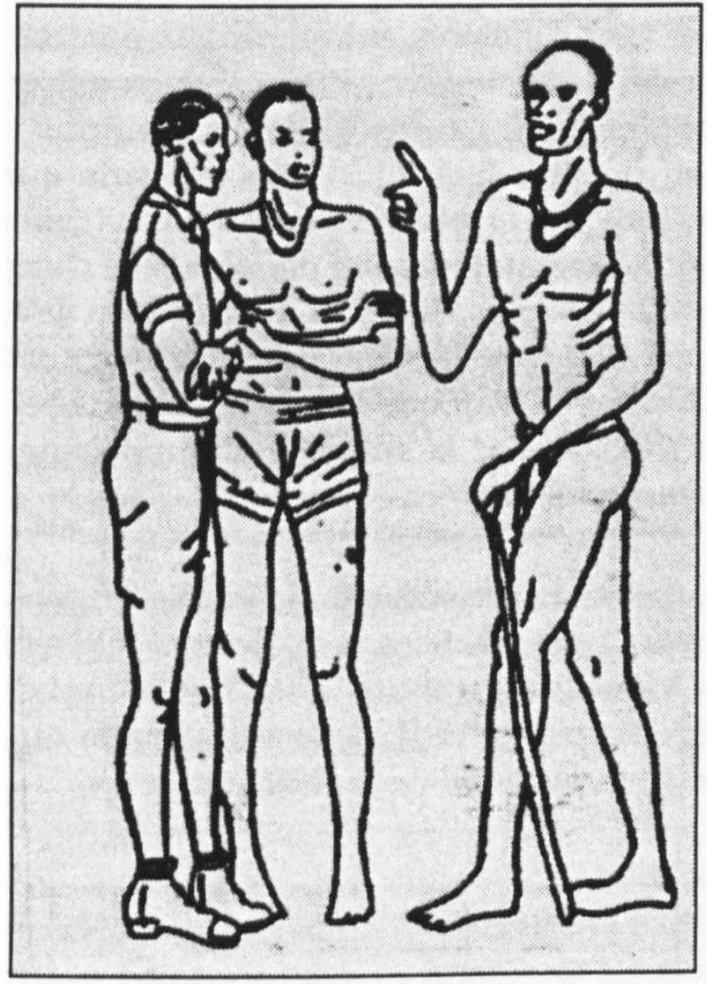

7 Ce qui fait la définition collective de la situation représentée par ce dessin c'est la configuration des éléments qui ont chacun une définition culturelle claire (nous soulignons ces éléments ci-après dans notre analyse). Remarquons que tous ces éléments nous sautent aux yeux en même temps. Nous les percevons ensemble, immédiatement et intuitivement. Ce n'est que par nécessité d'écriture que nous les décrivons, ci-dessous, un à un.

8 Les deux hommes plus jeunes sont côte à côte et se touchent même, cette proximité physique donne une indication sur leur relation « de personnes qui sont proches l'une de 
l'autre ». Ils ont les bras croisés, ce qui immanquablement signale leur non-activité, leur écoute ou leur attente. L'autre homme leur fait face et les regarde avec le doigt pointélevé et est assez proche d'eux, ceci indique qu'il leur parle. Le doigt pointé et levé indique le conseil ou la mise en garde. Le personnage de droite s'appuie sur une canne. La canne est éminemment le signe de la vieillesse. Les physionomies indiquent des âges différents entre les deux personnages de gauche et celui de droite, ceci, s'ajoutant aux autres significations données par les autres éléments, confirme l'idée de situation où un plus âgé, plus puissant parle à des plus jeunes. Le «sens » de la situation est alors : « situation où un plus âgé fait une communication dans laquelle il engage son autorité face à deux plus jeunes ». La vérification de cette perception signifiante a été faite sur de très nombreux sujets de diverses cultures, si bien que l'on peut même dire, pour ce dessin, qu'il représente la situation anthropologique de la « relation d'autorité ».

9 Si l'on met les sujets devant la situation figurée ci-dessous, on obtient des réactions radicalement différentes. Cela s'explique immédiatement par le fait que la forme de la situation représentée est radicalement différente du seul fait du changement d'un seul de ses éléments.
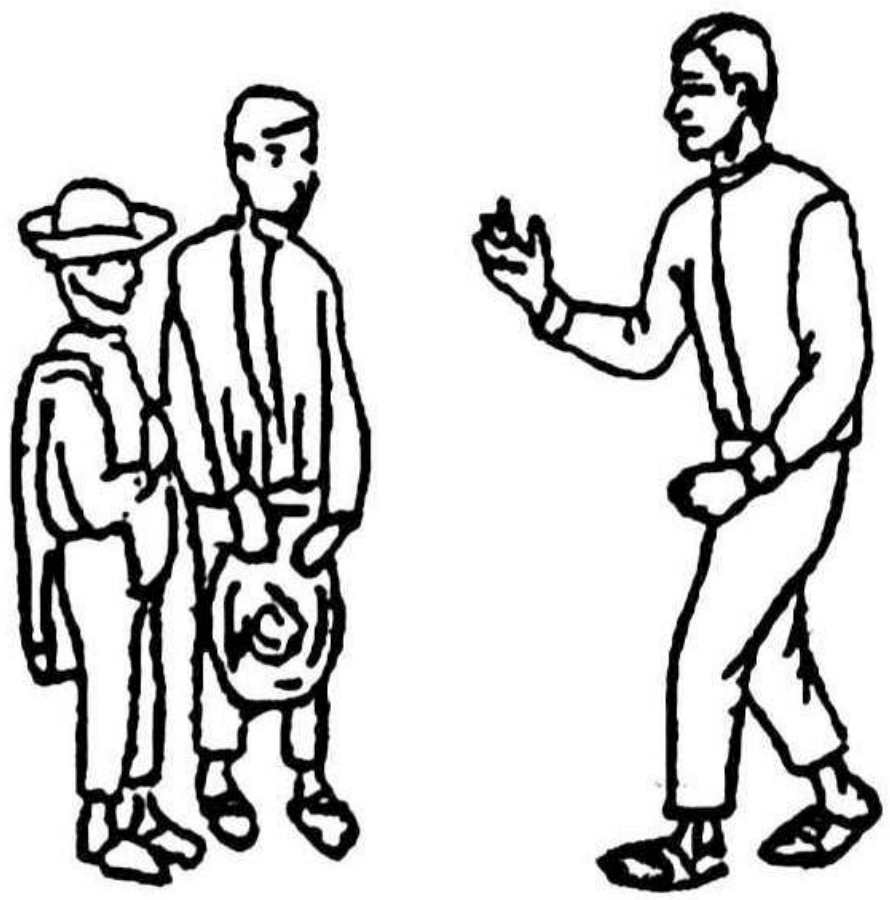

Le fait que le personnage "plus vieux» ait la main ouverte change le sens total qui devient soit «situation de sollicitation" (mendicité, $60 \%$ environ des interprétations), soit «situation de menace» (celui de droite va donner une gifle, $40 \%$ environ des interprétations).

11 En revanche si l'on met les sujets devant le graphique suivant, la situation évoquée est la même que pour la première planche vue ci-dessus. 


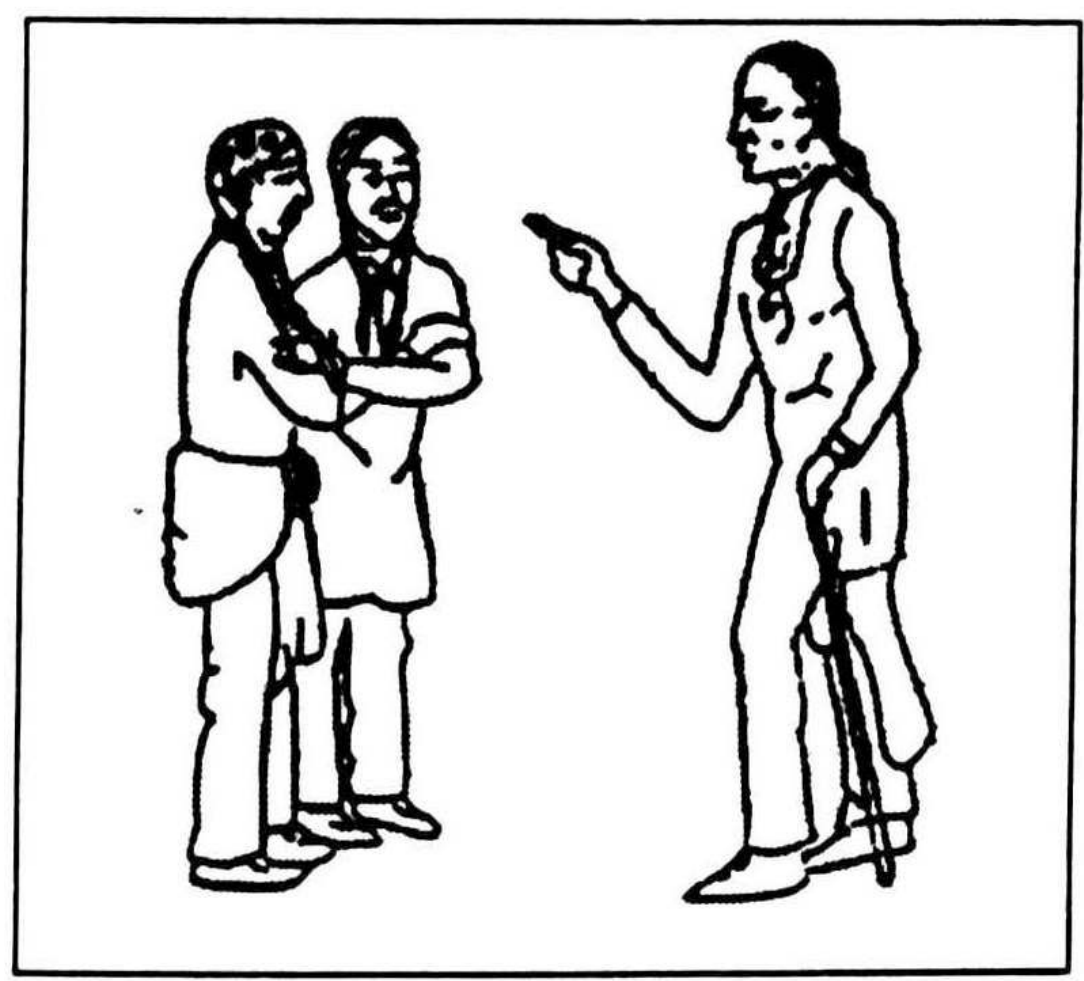

12 Ceci prouve, entre autres choses, que les éléments inducteurs constituant la « forme » de la situation sont des «abstraits». Ils fonctionnent en quelque sorte sous leur forme conceptuelle. Ce sont les éléments : " plus âgé », " remontrance-injonction », " deux plus jeunes » et " face à face » qui constituent la « situation de relation à l'autorité ». Mais, il est important de remarquer que cette "forme conceptuelle" contient tous les autres exemples concrets qui participent à la création de sa catégorisation. Ce sont tous ces exemples sociaux concrets qui sont convoqués lorsque le sujet se trouve en présence de l'élément. Ce sont eux qui influencent alors ses communications.

\section{Eléments inducteurs et normes déjà établies}

Pour illustrer l'influence des normes «établies » (les « déjà là » sociaux implicites) à travers certains éléments de la situation, je propose de considérer une expérience destinée à mettre en évidence ce phénomène. Cette expérience, comme toute expérience, peut être facilement refaite et ceci en fait son intérêt.

14 L'expérience citée ci-après, reprend l'idée du test de frustration de S. Rosenzwieg. Il s'agit d'une situation de testage. Le sujet doit se mettre à la place d'un des acteurs d'une situation imaginaire présentée sous la forme d'un dessin, et il doit indiquer ce qu'il dirait dans cette situation. Les sujets de l'expérience sont donc mis en présence d'un des deux dessins ci-dessous et on leur demande de dire ce qu'ils répondraient s'ils étaient à la place du conducteur admonesté. 


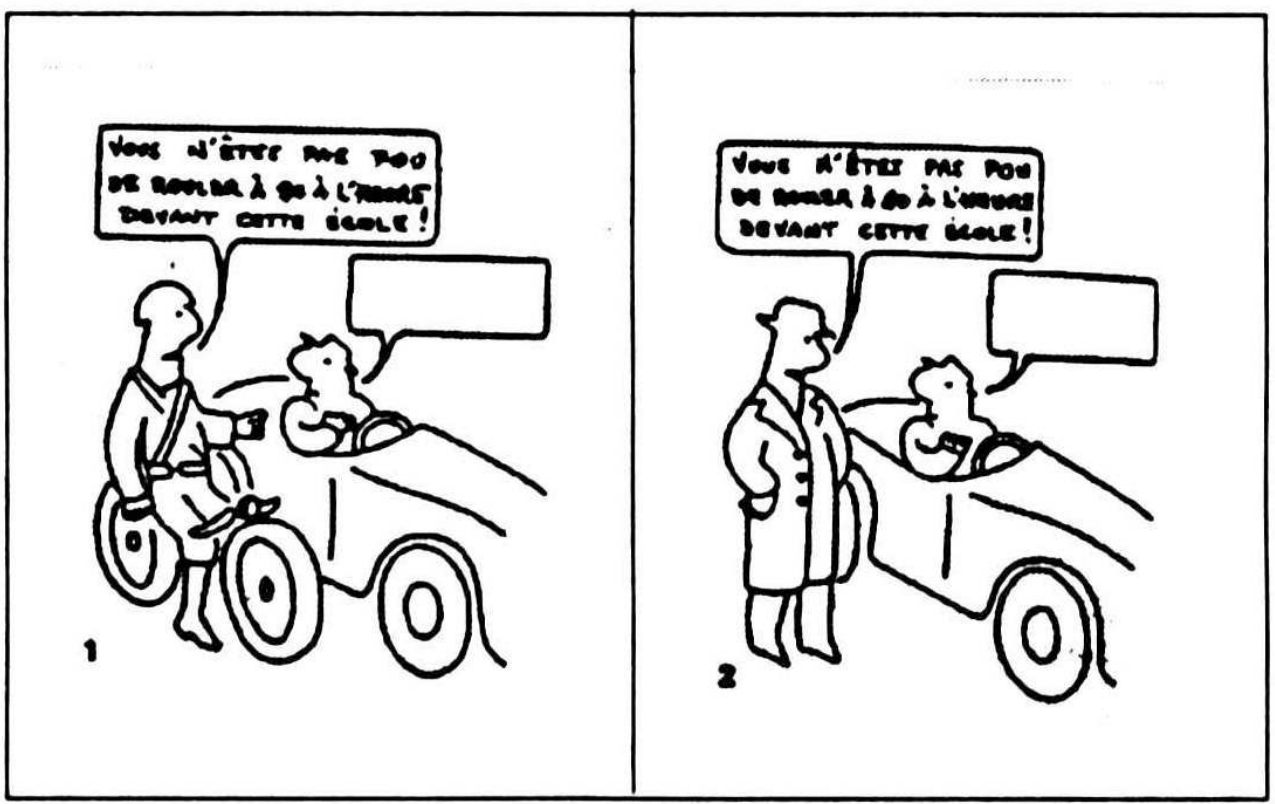

Voici, à quelques variations près, ce que l'on obtient habituellement sur des sujets occidentaux normalement socialisés.

Réponses obtenues à la situation avec le policier :

\begin{tabular}{|l|c|c|}
\hline \multirow{2}{*}{ Catégories des réponses } & \multicolumn{2}{|c|}{ Sujets } \\
\cline { 2 - 3 } & Hommes & Femmes \\
\hline $\begin{array}{l}\text { 1 - le sujet nie que sa culpabilité } \\
\text { soit entière en évoquant des } \\
\text { circonstances atténuantes }\end{array}$ & $58 \%$ & $50 \%$ \\
$\begin{array}{l}\text { - le sujet nie agressivement } \\
\text { être responsable de cette faute }\end{array}$ & $30 \%$ & $30 \%$ \\
3 - autres types de réponses & $12 \%$ & $20 \%$ \\
\hline
\end{tabular}


Réponses obtenues à la situation avec le piéton

\begin{tabular}{l|c|c}
\multirow{2}{*}{ Catégories des réponses } & \multicolumn{2}{|c}{ Sujets } \\
\cline { 2 - 3 } & Hommes & Femmes \\
\hline 1 - la culpabilité est admise & $19 \%$ & $9 \%$ \\
mais elle est minimisée & & \\
2 - négation agressive de la & $31 \%$ & $29 \%$ \\
culpabilité & $50 \%$ & $54 \%$ \\
3 - le sujet s'en prend au passant & - & $8 \%$ \\
4 - autres types de réponses & &
\end{tabular}

Les différences dans les réponses obtenues sont nettes. En présence du policier, la majorité des sujets admet sa culpabilité et essaie de la minimiser. En présence du piéton «tout venant ", la majorité des sujets (y compris les femmes) s'en prend directement à l'intervenant. La communication-réponse des sujets a donc été "orientée ». C'est ce phénomène « d'induction » qu'il nous faut analyser.

On voit immédiatement qu'un des éléments de la situation de «reproche" n'est pas le même entre la situation 1 et la situation 2. Le personnage qui fait le "reproche " n'a pas le même statut social. Dans un cas, il s'agit d'un motard, représentant le règlement, investi du droit de faire la réprimande verbale et du pouvoir de la faire suivre d'une contravention; dans l'autre cas, le simple passant «tout venant », ne représente plus du tout la même chose, et, on peut lui refuser le droit d'intervention dans la situation car il est celui qui « se mêle de ce qui ne le regarde pas ». Le «statut » de l'intervenant est donc « l'élément inducteur » immédiat.

Pour les sujets à qui l'on présente ces deux dessins, en leur demandant de se mettre à la place du conducteur, la situation n'est donc pas la même. L'élément «rôle social du réprimandeur » a changé et la totalité du sens de la situation a changé.

La présence ou l'absence du motard, sont en effet, des éléments fondamentaux qui donnent un sens à la situation, compte tenu des autres éléments qui ne changent pas. Lors de la présence du motard, la situation de reproche est une situation de «faute probable puisque reprochée par quelqu'un dont c'est le rôle social de repérer ce type d'infraction". C'est donc la «neutralité professionnelle » et «l'investiture sociale » du policier qui lui confèrent le «droit» d'intervenir. C'est ce sens social de la situation qui intervient pour «inhiber " les réponses de dénégation de la culpabilité ou les réponses d'agression défensive de l'automobiliste et qui « oriente » ses réponses vers des réponses de protection et de soumission. Ce «sens social » apparait immédiatement à tout adulte occidental normalement socialisé. On peut dire que la présence du policier « induit » une réponse culturelle banale : lorsqu'on se trouve devant un policier qui nous reproche une infraction, on essaie de protester de son innocence et de minimiser la faute (on ne l'a pas fait intentionnellement et ce n'est pas si grave que cela).

Par contre, lorsque le motard est remplacé par un «piéton tout venant », quelqu'un qui peut avoir d'autres motivations à son action d'intervention que celles professionnelles et donc neutres du policier et dont ce n'est pas le rôle social, les réponses d'agression 
défensive ne sont plus inhibées. En effet, le sens de la situation a changé et il est devenu : « situation dans laquelle un passant, sans investiture sociale, se met à jouer au policier en essayant par ce fait de culpabiliser l'automobiliste ». L'élément " piéton tout venant », par le biais de la restructuration du sens de la situation induit d'autres réponses: des réponses agressives où le sujet s'en prend au piéton («de quoi vous mélez-vous espèce de...»), et des réponses de dénégation (puisqu'il n'y a plus "d'objectivité professionnelle » de la part de l'interlocuteur) du genre: «pas du tout, vous n'avez pas bien apprécié !».

21 Nous voyons donc, sur cet exemple expérimental, comment, quelles que soient les personnalités - c'est-à-dire ici quels que soient les individus mis dans la situation de l'automobiliste qui se voit reprocher une faute - les réponses subissent l'influence $d u$ statut social du personnage réprimandant. C'est ce statut du « réprimandeur » qui change le sens de la situation. Ce statut de l'interlocuteur-motard ou piéton-est un «élément inducteur ». Il canalise les réponses des sujets dans deux directions bien distinctes : on s'excuse en minimisant sa faute ou on nie la faute lorsqu'il y a le policier; on minimise ou on agresse l'interpelant, dans l'autre cas. Mais, derrière ce statut social, ce sont les règles et les normes de communication régissant les situations sociales qui agissent. L'élément « inducteur » agit par le biais de la restructuration du sens de la situation à laquelle il participe en tant qu'élément du système des éléments définissant la situation. Ce sont, en dernière analyse, les normes comportementales sociales évoquées par la signification que prend la situation - à travers la présence d'un élément inducteur prépondérant-qui interviennent comme « éléments inducteurs cachés ».

\section{Éléments inducteurs et normes mises en place par les acteurs}

On connaît la fameuse enquête faite par Crozier dans une usine du monopole d'État de production de cigarettes. Cette enquête concerne les ateliers de production de ces usines. Une usine comporte plusieurs ateliers. Chaque atelier de production rassemble de trente à soixante machines de production. Le chef d'atelier dépend hiérarchiquement de la direction de la production de son usine. Un service d'entretien, sous la direction d'un ingénieur, fait des prestations aux différents ateliers de l'usine. Un groupe d'une douzaine d'ouvriers d'entretien est affecté à chaque atelier. Un ouvrier d'entretien a la charge de trois machines de l'atelier de production. 


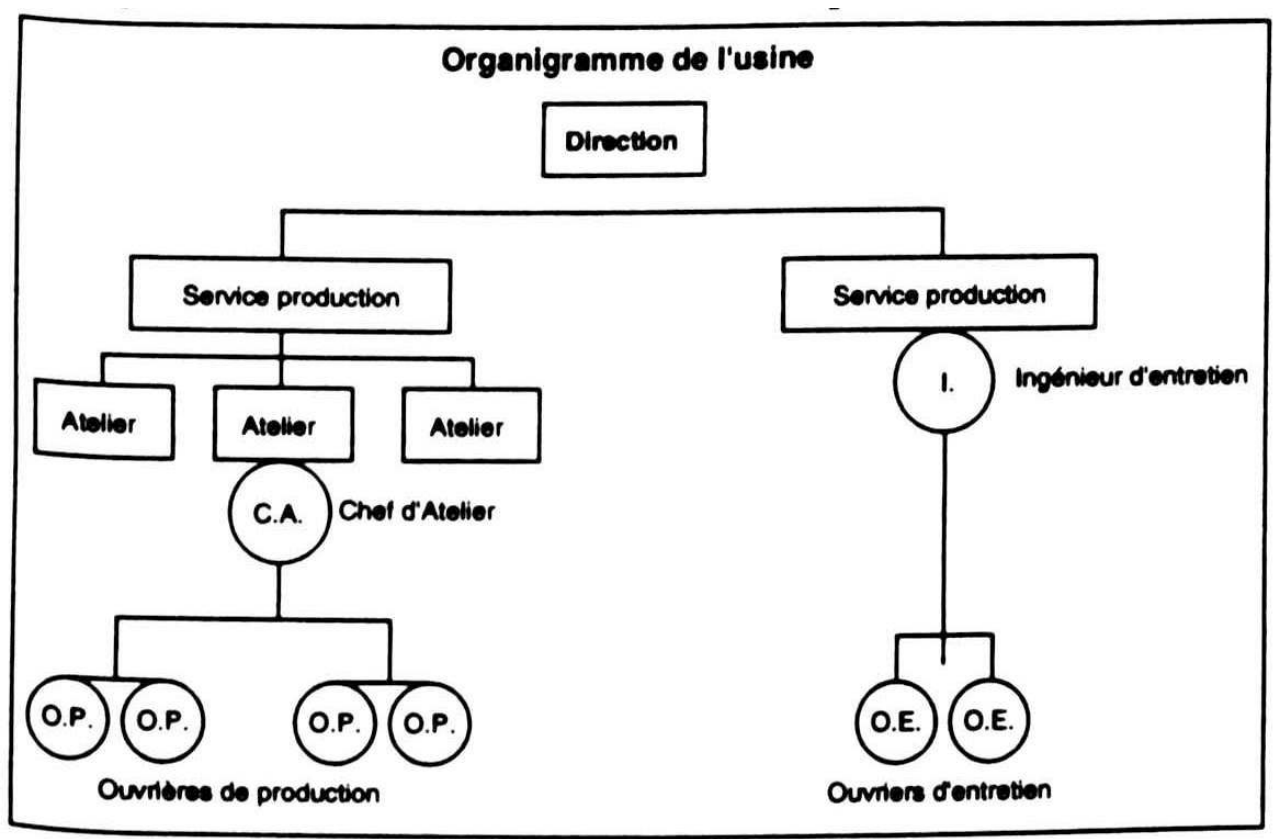
d'atelier, les ouvriers d'entretien et les ouvriers de production. L'ensemble des personnels jouit de la sécurité d'emploi attachée au statut de fonctionnaire. Chaque catégorie est recrutée par une filière séparée et il n'est pas prévu que l'on puisse, par examen ou promotion interne, passer d'une catégorie dans l'autre si bien que les statuts sont assez figés. Chaque catégorie de personnel a un rôle et des responsabilités bien définis dans le système de l'atelier de production.

Les chefs d'atelier s'occupent essentiellement de la gestion de la production: approvisionnement des machines des ouvriers, approvisionnement en fournitures diverses des ateliers, surveillance de l'utilisation des matières premières arrivant dans les ateliers, comptabilité de la production de l'atelier et de chaque poste de travail puisque les ouvriers sont payés au rendement. Ils ont en même temps un rôle de contremaître auprès des ouvriers de production; ils veillent, en particulier, à l'application du règlement lorsqu'il faut procéder à un remplacement sur un poste de travail. Le règlement est très précis : il est fondé sur la règle de l'ancienneté, le poste doit revenir à la personne volontaire, la plus ancienne en grade, s'il n'y a pas de volontaires, la personne la moins ancienne dans l'atelier est alors déplacée.

Les ouvriers d'entretien sont sous la responsabilité d'un ingénieur technique du service entretien de l'usine. Ce sont des ouvriers qualifiés munis d'un C.A.P. Ils ont chacun la charge, d'une manière permanente, de plusieurs machines de l'atelier de production et ils doivent entretenir et réparer ces machines. Ils sont une douzaine par atelier.

Les ouvriers de production sont en fait, en totalité, des ouvrières. Elles n'ont pas de niveau de qualification. Elles seraient O.S. dans l'industrie privée. Elles sont deux par machine : une conductrice de machine, une receveuse-manutentionnaire. Les affectations à ces postes de travail se font par l'application de la règle de l'ancienneté vue ci-dessus. Des normes de production sont établies d'une manière rationnelle et chaque équipe d'ouvrières touche des primes de rendement selon sa production.

Cette organisation du travail et cette distribution des rôles et des responsabilités font que le chef d'atelier a une autorité plutôt de prestige et de principe et que sa marge 
d'autonomie de décision est extrêmement limitée : il est recruté par une filière séparée (il est donc diplômé ou a des compétences précises), il est appelé « chef », il est responsable de toute l'administration de l'atelier, il contrôle a posteriori la production de chaque machine et détermine ainsi les attributions de primes, il fait appliquer la règle de pourvoiement des postes des ouvrières. Tout ce qu'il fait est réglementaire et prévu par des textes. Il n'a aucune marge de manœuvre dans l'appréciation des choses. Les ouvriers d'entretien sont sous la responsabilité, non pas du chef d'atelier, mais d'un ingénieur, lequel n'est pas directement en ligne hiérarchique sur l'atelier de production. Grâce à cet éloignement de leur responsable direct et au grand nombre de machines à entretenir, ils ont la liberté d'appréciation de l'opportunité de leurs interventions d'entretien. Ces interventions d'entretien puis de réparation lors des pannes sont capitales. Une machine bien entretenue ne tombe pas en panne et donc ses serveurs peuvent espérer des primes de rendement plus importantes. Lors d'une panne, ils sont certes, obligés d'aller réparer la machine, mais ils peuvent prétexter une intervention urgente prévenant une panne grave, sur une autre machine ou encore, ils peuvent faire durer leur intervention de réparation, pénalisant ainsi les ouvrières. Ils peuvent donc user, à leur gré, de leur savoir et de leur pouvoir d'intervention.

Cette organisation du travail et cette distribution des rôles et des responsabilités rendent complètement prévisibles les communications que peuvent entretenir les acteurs de cette situation de travail. La situation ainsi construite induit les communications entre les différents acteurs.

En ce qui concerne le chef d'atelier, le fait que tout soit prévu par le règlement dans ses relations de travail avec les ouvrières ne l'incite pas à développer d'autres échanges. Il ne pourrait que recevoir des rejets s'il se mêlait de vouloir développer des relations avec les ouvrières, en effet, celles-ci craindraient trop d'indisposer les ouvriers d'entretien dont elles dépendent (un jeune chef d'atelier s'étant d'ailleurs mélé de réparer une machine alors qu'un ouvrier d'entretien tardait à intervenir, fut déjugé par la direction et muté). On peut donc prévoir des relations "neutres " entre chefs d'atelier et ouvrières. Le contenu des communications est alors déterminé : il doit être limité aux banalités et aux civilités les plus courantes. Étant donné l'importance des ouvriers d'entretien aux yeux des ouvrières, leurs relations avec les ouvriers d'entretien ne peuvent être bonnes. Elles ne peuvent être que conflictuelles. Mais, dans le quotidien, étant donné le verrouillage des responsabilités, ils n'ont rien à gagner à entretenir le conflit ouvert et permanent. On peut donc prévoir que les chefs d'atelier essaieront de maintenir les ouvriers de production à distance et que leurs communications avec eux seront tout à fait restreintes.

En ce qui concerne les ouvriers d'entretien, étant donné les pouvoirs très limités du chef d'atelier - malgré son statut de "chef « - et compte tenu de l'importance de leurs interventions sur les machines pour les ouvrières, ils ne peuvent pas communiquer d'une manière amicale, déférente ou autoritaire avec les chefs d'atelier. Ils ne peuvent que communiquer d'une manière conflictuelle. On peut prévoir qu'ils doivent, par ailleurs, chercher à les dévaloriser aux yeux des ouvrières. On peut prévoir aussi que les ouvriers d'entretien vont avoir tendance à communiquer de manière autoritaire et condescendante avec les ouvrières de production qui sont sous leur dépendance en ce qui concerne leur travail et leurs primes de rendement.

31 En ce qui concerne les ouvrières de production, parce qu'elles sont sous la dépendance des ouvriers d'entretien, (et en retour de la communication autoritaire et condescendante qu'elles reçoivent d'eux), elles ne peuvent pas ne pas être tendues dans leurs relations 
avec ces ouvriers d'entretien. Par ailleurs, leur intérêt est d'être dans de bonnes relations avec l'ouvrier d'entretien qui s'occupe de leur machine. Les ouvrières ne peuvent pas communiquer d'une manière intense avec les chefs d'atelier car ceux-ci ne sont pas perçus comme des personnages importants pouvant intervenir sur leur travail (et cela pourrait indisposer leur ouvrier d'entretien). Elles n'ont donc pas d'intérêt à communiquer avec leur chef d'atelier. Le mieux, pour elles, c'est d'avoir avec eux des relations cordialement neutres.

Ces prévisions, fondées sur l'analyse des enjeux créés par l'organisation du travail pour les différents acteurs, a été entièrement validée par l'enquête de Crozier. C'est ainsi que l'on peut dire que la situation définit, pour chacun, « ce qu'il faut faire » pour s'en sortir au mieux (c'est-à-dire sauvegarder ses intérêts). La situation de travail construite par les hommes contient donc des « règles » que les acteurs suivront. La situation construite agit par l'intermédiaire de ces normes qui apparaissent, en dernier ressort, comme les véritables « éléments inducteurs ".

\section{Éléments inducteurs et normes émergentes à travers les échanges}

33 On connaît les très célèbres expériences de $\mathrm{K}$. Lewin sur les différents rôles de leaderschip. Lewin a défini trois rôles précis : les rôles des animateurs démocratiques, autocratiques et laisser-faire. Ces rôles sont définis par des attitudes et par une série de conduites. Les expériences d'animation ont été faites sur des groupes de jeunes adolescents envoyés par leurs parents dans un club d'aéromodélisme payant pour se distraire et se former.

Le rôle de l'animateur « autocratique » était ainsi défini : il garde pour lui l'objectif final du travail du groupe (ne montre pas la maquette finale), il décide seul de l'attribution des tâches qu'il distribue, dicte à chacun, pas à pas, ce qu'il doit faire sans donner une vue d'ensemble de la tâche ni préciser comment elle s'inscrit dans le travail global, il s'adresse individuellement aux membres du groupe sans jamais employer de "nous » collectif, il communique uniquement des ordres, des gratifications ou des réprimandes et, enfin, il ne participe pas lui-même à la réalisation de la maquette.

Ce «style de leaderschip ", c'est-à-dire ces communications lors du travail du groupe, crée de toute pièce - et ceci indépendamment des personnalités des acteurs du groupedes phénomènes de caractéristiques: il $\mathrm{y}$ a donc un phénomène d'induction. Les principaux phénomènes toujours constatés sont les suivants : les adolescents s'adressent exclusivement à l'animateur, ils ne se parlent pas entre eux, ils demandent des instructions (ils ne montrent aucune initiative et créativité), ils quémandent ses gratifications, ils se plaignent de leurs camarades (ils sont donc dépendants à l'égard du leader), ils se disputent et se chamaillent, un esprit de compétition se développe, un certaine tension règne, les adolescents, à la fin du travail ne sont pas contents de leur maquette et jugent l'expérience peu intéressante...

Examinons sur quoi sont fondées les inductions dans le cas du leader autocratique.

L'animateur autocratique par ses attitudes et ses conduites crée des normes de conduites que les adolescents - prisonniers de la situation - suivent. En effet, ses comportements signifient aux membres du groupe que seul compte pour lui son rôle de chef, que seul compte le résultat à atteindre, et donc, que, le groupe, le travail collectif, l'ambiance, 
n'ont aucune importance. En gardant pour lui l'objet final du travail et en dictant pas à pas ce qu'il faut faire, il signifie que la réalisation de la tâche est sa seule affaire, que les participants ne sont que des exécutants de ses ordres; il dit donc implicitement que chacun n'a pas à s'impliquer dans la réalisation finale. Il est bien normal alors, qu'au bout du compte, les acteurs ne se sentent pas impliqués dans la production commune, jugent leurs tâches peu intéressantes et soient peu satisfaits du travail final. L'animateur autocratique distribue sans concertation les sous-rôles à tenir dans le groupe, il s'adresse individuellement à chacun, n'emploie jamais le « nous » collectif, et donne des indications précises. Il signifie donc par cet ensemble de comportements que la collectivité n'a aucune valeur et que le groupe n'est pas le lieu d'une coopération; il dit donc implicitement qu'il n'y a pas à entretenir de relations interindividuelles (amicales, de coopération, d'émulation, de création commune...). L'animateur autocratique, enfin, ordonne des actions, félicite ou réprimande individuellement les membres de son groupe. D signifie par là qu'il est la seule source de la marche du groupe et qu'il n'y a pas d'autres critères d'évaluation des comportements. Il dit donc implicitement: "référez-vous seulement à moi », ce qui induit la dépendance des membres par rapport à lui, et : « tout ce qui n'est pas travail est sans valeur " (ou, autre formulation de la même injonction implicite : «les sentiments n'ont pas cours ici »), ce qui coupe toute possibilité d'échange amical entre les membres.

Finalement toutes les communications de l'animateur sont porteuses de normes. Ceci, comme nous l'avons déjà dit, parce que la situation est fermée et n'offre pas d'autres chemins aux comportements. Peut-on penser à une révolte d'un de ces adolescents contre l'adulte-animateur fourni par le club de loisirs auquel il est inscrit par ses parents ? Cette révolte ne peut être effective. Elle est latente chez ceux qui acceptent le moins le rôle obligé de suiveur-dépendant. Elle contribue à faire monter la tension ce qui rend encore plus malaisées la coopération, les relations amicales, l'invention créative par le groupe et la satisfaction envers le travail accompli. Peut-on penser à une contestation introduite par un contre-leader émergeant du groupe ? Peut-on penser à l'apathie réactionnelle de tous les acteurs ou à leur fuite de la situation? Pas davantage étant donné les contraintes morales de la situation et les pressions exercées par l'animateur.

Cet ensemble de règles donne le sens global de la situation de communication : « situation de réalisation d'un travail sous la contrainte morale d'un animateur autoritaire dont le statut n'est pas discutable». C'est ce sens global, renvoyant à la situation scolaire traditionnelle, et appelant les normes comportementales qui lui sont attachées qui « induit » largement les communications des adolescents.

\section{Conclusion}

40 Le phénomène d'induction des communications que l'on peut constater dans une certain nombre de situations d'échanges entre des acteurs, se réalise à travers l'importance prise, pour ces acteurs, par certains éléments du contexte présent. Ces derniers éléments sont perçus comme significatifs parce que représentatifs d'un ensemble de situations sociales qui sont ainsi évoquées. Cette évocation appelle automatiquement les normes sociales attachées à ces situations. Ce sont alors ces normes sociales qui interviennent, en dernier ressort, sur les communications des acteurs.

41 Ainsi, les normes sociales s'avèrent avoir un poids important dans les phénomènes d'induction des communications. Ceci renforce toutes les analyses faites pour 
décomposer la communication en "processus internes » et pour mettre en évidence le "processus de construction des référents collectifs» (les règles que doivent suivre les échanges). Cette construction des référents collectifs à travers la communication peut donc se faire de plusieurs manières: par appel à des normes déjà établies, par conformisation à des règles implicites mises en place par les acteurs, par construction de normes relationnelles au cours des échanges eux-mêmes.

\section{BIBLIOGRAPHIE}

CROZIER M., Le phénomène bureaucratique, Seuil, 1963, pp. 70-174.

LEWIN K., LIPITT R., WHITE R.K., Patterns of agressive behavior in experimental created social climate, J. Soc. Psychol., X, 271, p. 299,1939.

MUCCHIELLI A., Les situations de communication, Eyrolles, 1991, pp. 86-89.

MUCCHIELLI A., Les sciences de l'information et de la communication, Hachette, 1996, chapitre : « Les processus de la communication », pp. 113-150.

VILLERBU L., Examen critique du « Picture frustration study test » de S. Rosenzwieg, Thèse pour le doctorat ès Lettres, Nice, 1969.

\section{AUTEUR}

\section{ALEX MUCCHIELLI}

Université Paul Valéry-Montpellier III, Centre de Recherche sur l'Information et la Communication 